\title{
Evaluation de la protection personnelle contre les Anophelinae par utilisation de moustiquaires détériorées imprégnées de répulsifs
}

\author{
Athanase BADOLO ${ }^{1,2^{*}}$, Edith ILBOUDO-SANOGO ${ }^{2}$, Antoine SANON ${ }^{1}$ and \\ Albert Patoin OUÉDRAOGO ${ }^{1}$ \\ ${ }^{1}$ Université de Ouagadougou, BP 7021 Ouagadougou 03, Burkina Faso. \\ ${ }^{2}$ Centre National de Recherche et de Formation sur le Paludisme, (CNRFP) 01 BP 2208 Ouagadougou 01, \\ Burkina Faso. \\ *Auteur correspondant; E-mail : a.badolo@gmail.com; Tel: + 2265032 46 95/6, Fax: + 22650305220
}

\section{RESUME}

L'efficacité de moustiquaires détériorées imprégnées avec des répulsifs (DEET et KBR 3023) ou avec la perméthrine a été évaluée dans la protection contre Anopheles gambiae s.l. L'étude a été conduite dans le village de Goden près de Ouagadougou. Les moustiquaires ont été installées dans des cases sélectionnées dans le village et les moustiques ont été échantillonnés à l'aide de pièges lumineux CDC, de captures manuelles et de pièges à sortie. Les résultats montrent que le nombre de moustiques capturé ainsi que leur état physiologique varie selon le traitement et le degré de détérioration de la moustiquaire. La réduction de l'agressivité exprimée par les moustiques gorgés est plus importante avec le DEET et le KBR 3023 qu'avec la perméthrine (57\%, $76 \%$ et $43 \%$ respectivement). Ce résultat est observé pour une surface de détérioration égale à $0,1 \%$ et une tendance similaire est observée à $1 \%$ de surface de détérioration. L'efficacité des moustiquaires varie en fonction de la case, de la surface de détérioration et du temps après imprégnation de la moustiquaire. Le KBR 3023 procure la meilleure protection par rapport aux autres traitements et même par rapport à la moustiquaire intacte lorsque la surface de détérioration est faible $(0,1 \%)$. L'utilisation des répulsifs en imprégnation pourrait accroître l'efficacité des moustiquaires usagées.

() 2012 International Formulae Group. All rights reserved.

Mots clés : DEET, KBR 3023, Perméthrine, fraction agressive, rémanence, moustiquaires endommagées.

\section{INTRODUCTION}

La stratégie actuelle de l'OMS en matière de lutte antivectorielle repose essentiellement sur les moustiquaires imprégnées et les pulvérisations intradomiciliaires d'insecticides (Nabarro et Tayler, 1998).

De nombreuses études ont montré que l'utilisation au niveau communautaire de matériaux imprégnés d'insecticides pyréthrinoïdes permettait une réduction de la morbidité et de la mortalité palustres (Enayati et Hemingway, 2010; Lengeler, 2004). Ils influencent les paramètres entomologiques du paludisme tels que le nombre de piqûres d'anophèles reçues (Robert et Carnevale, 1991), le taux d'inoculation entomologique (Ilboudo-Sanogo et al., 2001) . L'apparition et l'expansion de la résistance aux pyréthrinoïdes dans les populations ouestafricaines de Anopheles gambiae (Chandre et al., 1999; Ranson et al., 2009) constituent une 
menace pour cette stratégie de lutte (N'Guessan et al., 2007; Sharp et al., 2007).

Les moustiquaires en parfait état non imprégnées peuvent protéger à $90 \%$ contre les piqûres de moustiques (Snow et al., 1988). Dans les zones rurales d'Afrique, les moustiquaires utilisées sont souvent endommagées (Macintyre et al., 2011), ce qui augmente le risque de transmission du paludisme par rapport à une moustiquaire intacte (Carnevale et al., 1992). Un argument en faveur de l'imprégnation des moustiquaires avec les insecticides pyréthrinoïdes est leur capacité à restaurer l'efficacité des moustiquaires même usagées (Carnevale et al., 1992; Lines, 1996). Ceci permet de réduire le nombre de moustiques qui pénètrent à l'intérieur des moustiquaires endommagées ou qui piquent à travers les mailles des moustiquaires intactes.

Des études de laboratoire et de terrain ont montré une efficacité relative du KBR 3023 au moins égale a celle du DEET vis-àvis d'An. gambiae s.l. et d'Aedes aegypti (Badolo et al., 2004; Costantini et al., 2004). Le DEET est reconnu comme répulsif de référence (Frances et al., 1998; Qiu et al., 1998; Rutledge et al., 1983). Le problème commun aux répulsifs de façon générale et au DEET en particulier est leur faible persistance (Curtis et al., 1987), ce qui nécessite une application cutanée régulière, posant un problème de coût. Il faut ajouter à ces problèmes, les propriétés anti-plastiques, les considérations d'ordre esthétique et le risque de réactions allergiques cutanées et de toxicité (Corbel et al., 2009; Qiu et al., 1998). Ce sont essentiellement ces raisons qui entravent l'utilisation des répulsifs en application topique dans la protection contre les piqûres des insectes vecteurs de maladies comme le paludisme. Une solution pourrait être l'utilisation des répulsifs en imprégnation sur matériaux (Curtis et al., 1987; Pennetier et al., 2009). En effet, l'imprégnation sur matériaux pourrait accroître la rémanence du répulsif du fait de l'adsorbance plus grande des tissus par rapport au corps humain et réduire ainsi le contact des répulsifs avec le corps des utilisateurs (Rozendaal, 1997).

Ce sont ces différentes raisons qui ont motivé notre étude sur l'évaluation de l'efficacité des moustiquaires imprégnées de répulsifs (DEET et KBR 3023) et de la perméthrine contre Anopheles gambiae s.l. L'objectif poursuivi par cette étude étant d'évaluer la capacité des répulsifs DEET et KBR 3023 à restaurer l'efficacité de moustiquaires détériorées. De façon spécifique, nous avons évalué l'influence du temps et du degré de détérioration des moustiquaires sur la protection qu'elles procurent contre les moustiques Anopheles gambiae s.1., vecteurs du paludisme.

\section{MATERIEL ET METHODES}

\section{La zone d'étude}

L'étude s'est déroulée en zone rurale dans le village de Goden $\left(12^{\circ} 25^{\prime} \mathrm{N}, 1^{\circ} 20^{\prime} \mathrm{W}\right)$, situé à $30 \mathrm{~km}$ à l'Est de la ville de Ouagadougou, Burkina Faso. Les vecteurs du paludisme dans cette zone appartiennent au complexe An. gambiae s.l. dont on rencontre trois taxa: An. arabiensis et les formes moléculaires $\mathrm{M}$ et $\mathrm{S}$ de An. gambiae s.s (Fanello et al., 1999).

\section{Traitement des moustiquaires}

Douze moustiquaires en coton de dimensions $1 \mathrm{~m}$ x $2 \mathrm{~m}$ x 1,5 m (largeur x longueur $\mathrm{x}$ hauteur) ont été utilisées. Sur les côtés des moustiquaires, 110 trous de dimensions variables $\left(1 \mathrm{~cm}^{2}, 10 \mathrm{~cm}^{2}\right.$, et 100 $\mathrm{cm}^{2}$ ) ont été répartis de façon régulière sur la surface de la moustiquaire pour obtenir trois lots de moustiquaires avec une surface endommagée de $0,1 \% ; 1 \%$ et $10 \%$ respectivement. Ces moustiquaires ont été imprégnées avec des émulsions en eau de formulations "technical grade" soit des répulsifs de synthèse DEET et KBR 3023 à 10 $\mathrm{g} / \mathrm{m}^{2}$ de matière active, soit avec de la perméthrine (Imperator ${ }^{\mathrm{TM}} 50 \mathrm{EC}$ ) à la dose cible de $0,5 \mathrm{~g} / \mathrm{m}^{2}$ ou avec de l'eau en tant que témoin. La quantité d'eau utilisée a été déterminée par trempage suivant la méthode exposée dans Chavasse et al. (1999), qui tient 
compte de l'adsorbance de chaque moustiquaire. Les moustiquaires imprégnées sont séchées à l'air libre et conditionnées dans des cartons jusqu'au jour de l'utilisation, soit deux jours après l'imprégnation.

\section{Protocole expérimental}

Vingt et quatre cases ont été choisies pour $\mathrm{y}$ effectuer les captures. Le critère de choix incluait :

- La présence dans la case de deux ouvertures en plus de l'entrée principale pouvant permettre d'installer un piège à sortie, l'autre entrée pouvant laisser entrer les moustiques.

- La case devait être habitée par une seule personne (souvent le chef de concession) ceci dans le but de ne pas perturber les habitudes de couchage des populations, puisque la capture devait se faire auprès d'une seule personne dormant sous la moustiquaire.

- L'occupant de la case ne dormait pas initialement sous moustiquaire pour ne pas exposer la personne à plus de piqûres qu'elle n'en recevait initialement.

Les cases ont été appariées suivant leurs densités de moustiques proches sur la base des captures préliminaires. Dans chaque paire, les traitements étaient identiques. Pour limiter l'effet de contamination des répulsifs et de l'insecticide, les captures dans les cases d'une même paire se faisaient de façon alternée.

Les captures ont été étalées sur une période de trois mois en répliquant des carrés latins $12 \times 12$ (jours x cases), avec une rotation aléatoire des traitements entre les cases au sein du même carré latin, quatre carrés latins ont été réalisés en tout. Afin de suivre la dynamique de la population d'An. gambiae s.l. au cours de l'expérience, une moustiquaire intacte non imprégnée a été installée dans une case sentinelle supplémentaire dotée des mêmes outils d'échantillonnage que les cases expérimentales.

\section{Méthodes d'échantillonnage et manipulation}

Pour évaluer le nombre de moustiques qui entraient dans les cases et se gorgeaient sur les dormeurs volontaires, trois méthodes d'échantillonnage ont été mises en œuvre durant chaque nuit de capture, de $20 \mathrm{~h}$ au réveil des volontaires à $5 \mathrm{~h}$ le lendemain. Un piège lumineux de type CDC était placé à coté de la moustiquaire et actionné comme décrit dans Costantini et al. (1996). Un piège de sortie (PS) constitué par une grande cage avec une embouchure en forme d'entonnoir était placé sur la façade externe d'une des deux fenêtres de chaque case pour recueillir les moustiques sortant de la case. Les moustiques qui au cours de la nuit ont pu rentrer à l'intérieur des moustiquaires endommagées étaient récoltés le lendemain matin à l'aide d'un aspirateur sur la surface interne de la moustiquaire pour les vivants et sur le lit pour les moustiques morts.

Les moustiques capturés étaient transférés dans des verres en carton, nourris avec du coton imbibé d'eau glucosée à $5 \%$, et transportés au laboratoire pour y être identifiés à l'aide de la clé de Edwards (1941) pour les Culicinae, de celle de Gillies et Coetzee (1987) pour les Anophelinae. Après identification, les moustiques sont conditionnés dans des tubes eppendorf contenant du gel de silice au regard de leur espèce et leur état gonotrophique.

\section{Analyse statistique}

Seuls les moustiques du complexe $A n$. gambiae s.l. ont été pris en compte pour l'analyse car les autres espèces étaient très faiblement représentées dans nos collectes. Pour une meilleure interprétation des résultats, l'échantillon a été subdivisé en trois fractions selon la méthode d'échantillonnage et l'état gonotrophique des moustiques capturés. Le nombre total de moustiques agressifs qui sont rentrés dans les cases au cours de la nuit a été estimé par le nombre total de moustiques capturés par les différentes méthodes en soustrayant les moustiques gravides dont on estime qu'ils ne venaient pas se gorger sur le dormeur. La fraction formée par tous les moustiques gorgés et partiellement gorgés durant cette nuit de capture a été considérée comme représentative des moustiques qui se 
sont gorgés sur le dormeur ou sur des hôtes à l'extérieur. Les moustiques gorgés capturés sous la moustiquaire représentent la fraction ayant franchi la moustiquaire pour se gorger sur le dormeur volontaire. Le nombre $x \mathrm{du}$ nombre de moustiques de chacune de ces fractions n'est pas distribué selon une loi normale. Par conséquent, nous avons transformé les données en $(\log (x+1))$, et nous avons exprimé les tendances centrales par la moyenne géométrique de William $M G$ qui représente la transformation inverse de la moyenne arithmétique des données transformées. Cela se traduit par la formule suivante:

$M G=\exp \left[\frac{1}{n} \sum_{i=1}^{n} \log \left(1+x_{i}\right)\right]-1$

Les intervalles de confiance de ces moyennes ont été calculés pour chaque traitement, surface de détérioration et pour chaque fraction de notre échantillon.

La signification statistique des différences entre les moyennes géométriques a été évaluée par une analyse de la variance (ANOVA). Puisqu'une des sources possibles de variabilité était la concession de capture, celles-ci ont été traitées comme des blocs dans une ANOVA à deux voies de mesures répétées. Nous avons calculé la moyenne par concession par unité de temps (Carré Latin) et par surface de détérioration. Les ANOVA se sont faites en deux étapes: D'abord en comparant l'effet moyen des traitements par rapport à la surface de détérioration sur l'ensemble de l'expérience sans tenir compte de la variable temps. Ensuite, en évaluant la réduction dans le temps de l'effet des traitements, sans tenir compte de la surface de détérioration. Les analyses ont été réalisées à l'aide du logiciel GLIM (Payne, 1987).

\section{Considérations éthiques}

Les volontaires ont été choisis sur la base qu'ils n'utilisaient pas initialement de moustiquaires pour ne pas les exposer à plus de piqûres qu'ils n'ont l'habitude d'en recevoir. Ils ont été bien informés des objectifs et méthodes de l'étude et ont été retenus comme dormeurs volontaires après qu'ils aient signé un consentement éclairé écrit. Ils n'ont pas changé de case du début à la fin de l'étude et n'ont pas changé leurs habitudes. Ainsi, ils n'étaient pas exposés à un niveau inhabituel de transmission du paludisme. Ils ont en outre subi un traitement prophylactique à la sulfadoxine pyriméthamine, et ont été suivis au plan clinique durant toute la période de l'étude. Un diagnostic parasitologique était posé en cas de fièvre, et un traitement leur était administré en cas d'accès palustre.

\section{RESULTATS \\ Dynamique de la population}

L'évolution du nombre total de $A n$. gambiae s.l. capturé chaque jour posttraitement dans la case sentinelle exprime la variabilité de densités de la population agressive au cours de l'essai ainsi qu'au sein de chaque Carré Latin (Figure 1a). Les densités journalières ont oscillé autour d'une valeur de 100 moustiques par jour pendant le premier et le dernier Carré Latin, et autour d'une valeur inférieure dans les deux Carrés Latins intermédiaires. Néanmoins, au sein de chaque Carré Latin et à l'exception du troisième, les densités moyennes sont restées assez stables (absence de forte tendance à la hausse ou à la baisse).

\section{Distribution des fractions selon la méthode d'échantillonnage}

La part des pièges lumineux CDC dans l'échantillon total de moustiques est la plus importante (entre 77 et $98 \%$ ) du nombre total de moustiques capturés par les différents pièges. Cette contribution des pièges CDC varie selon la surface endommagée de la moustiquaire, elle est faible lorsque la moustiquaire est détériorée à $1 \%$, tandis qu'elle est élevée à $0,1 \%$ et $10 \%$. A l'inverse, la fraction de moustiques sous la moustiquaire est élevée à $1 \%(22 \%)$ et faible à $0,1 \%$ et $10 \%$ 
(6\% et $8 \%$ respectivement). La part des pièges à sortie est la plus faible et varie de 1 à $2 \%$ (Tableau 1).

La proportion de moustiques gorgés est en moyenne plus faible avec les pièges CDC qu'avec les autres types de pièges à savoir le piège de sortie et la moustiquaire. La proportion de gorgés varie aussi avec la surface de détérioration de la moustiquaire, elle est plus importante avec les moustiquaires détériorées à $0,1 \%$ (Tableau 1 ).

\section{Efficacité des traitements}

Sur la fraction totale de l'échantillon, le nombre moyen de moustiques capturés est significativement plus élevé avec la perméthrine et faible avec le DEET et le KBR 3023. La différence entre DEET et KBR 3023 n'étant significative qu'à la surface détériorée de $0,1 \%$. La réduction des moyennes géométriques du nombre de moustiques capturés par rapport au témoin est plus élevée avec le KBR 3023 quelle que soit la surface de la moustiquaire considérée. Elle est par contre plus faible avec la perméthrine. En général, la réduction du nombre moyen de moustiques est faible lorsque la surface de détérioration de la moustiquaire augmente (Tableau 2).

$\mathrm{Au}$ niveau de la fraction gorgée de moustiques, le nombre moyen de moustiques est plus faible avec le KBR 3023 qu'avec le DEET et la perméthrine quelle que soit la surface de détérioration considérée. La réduction par rapport au témoin du nombre moyen de moustiques gorgés est plus élevée lorsque la surface de détérioration est de $1 \%$.

Le nombre moyen de moustiques capturés sous la moustiquaire est quasi nul avec la perméthrine, avec une réduction de $100 \%$ du nombre de moustiques par rapport au témoin.

\section{Effet de la surface de détérioration}

Le nombre total ainsi que la moyenne géométrique (MG) du nombre de moustiques capturés diminue par rapport aux traitements témoins lorsque la surface de détérioration de la moustiquaire augmente. Il n'y a pas de différence significative entre les MG dans la fraction totale de l'échantillon pour les surfaces détériorées de $0,1 \%$ et $1 \%$. En ce qui concerne la fraction gorgée et la fraction sous la moustiquaire par contre, les MG sont significativement plus élevées pour la surface de $1 \%$ (Tableau 2). La comparaison des moyennes par analyse de la variance montre que la différence est significative entre surfaces endommagées, traitements et entre cases $(P<0,01)$ en ce qui concerne les deux premières fractions de l'échantillon. Par contre la case n'a aucun effet $(\mathrm{P}>0,05)$ sur le nombre moyen de moustiques qui entrent dans la moustiquaire.

\section{Influence du temps après imprégnation.}

La perte d'efficacité des traitements dans le temps a été évaluée en calculant le rapport entre le nombre de moustiques capturés avec les traitements par rapport au témoin sur l'ensemble des captures au sein de chaque Carré Latin (Figures 1b, 1c, 1d). Les rapports sont restés inférieurs à $20 \%$ durant le premier mois post-traitement pour les deux répulsifs (DEET et KBR 3023) pour la fraction totale, et égales ou proches de $0 \%$ pour les captures à l'intérieur de la moustiquaire. Ces valeurs ont varié entre $20 \%$ et $60 \%$ durant le dernier Carré Latin (Plus de 2 mois après l'imprégnation) en rapport avec la fraction de l'échantillon et le répulsif considérés. Un pic dans les rapports a été atteint pour tous les traitements et toutes les fractions pendant le troisième Carré Latin, correspondant à une période de baisse importante des densités de An. gambiae s.l. dans le village (Figure 1a). Cette observation suggère que l'effet des traitements est densité dépendant. La rémanence due au KBR 3023 a été toujours plus grande que celle du DEET pour les trois fractions.

Les résultats obtenus avec la perméthrine ont été plus variables. Si d'une 
part les rapports ont oscillé autour de l'unité pour la fraction totale et la fraction de moustiques gorgés (avec une discontinuité entre les deux premiers et les deux derniers Carrés Latins), ils sont restés constamment proches de $0 \%$ pour la fraction capturée à l'intérieur de la moustiquaire pendant la durée de l'expérience.

L'analyse de la variance montre que

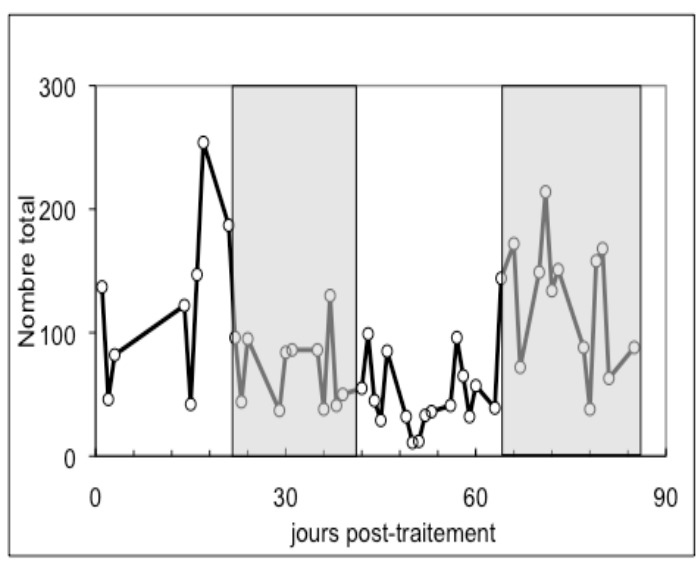

a

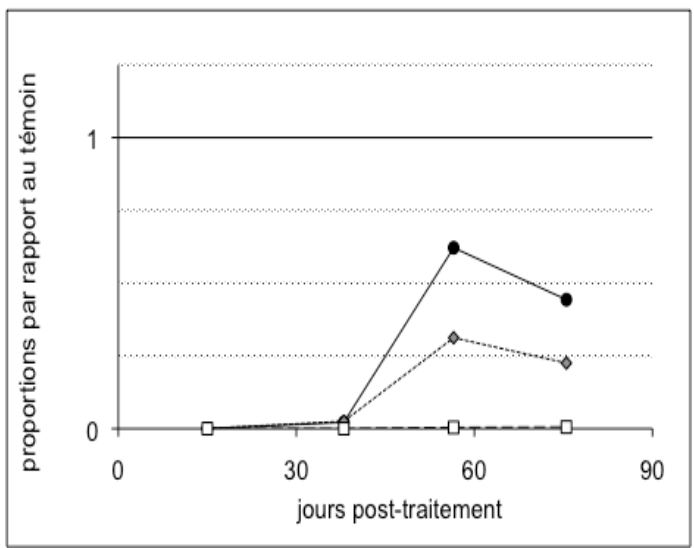

C le temps après imprégnation, la case où a lieu la capture, le traitement et l'interaction traitement $\mathrm{X}$ temps ont un effet statistiquement significatif $(\mathrm{P}<0,05)$ sur le nombre moyen de moustiques capturés pour la fraction totale, la fraction gorgée et la fraction sous la moustiquaire de l'échantillon total, l'effet de la case étant non significatif au niveau de la fraction sous moustiquaire.

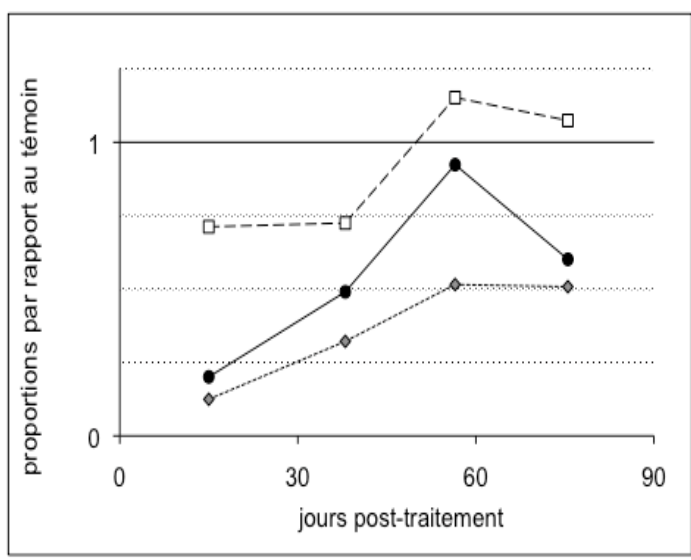

b

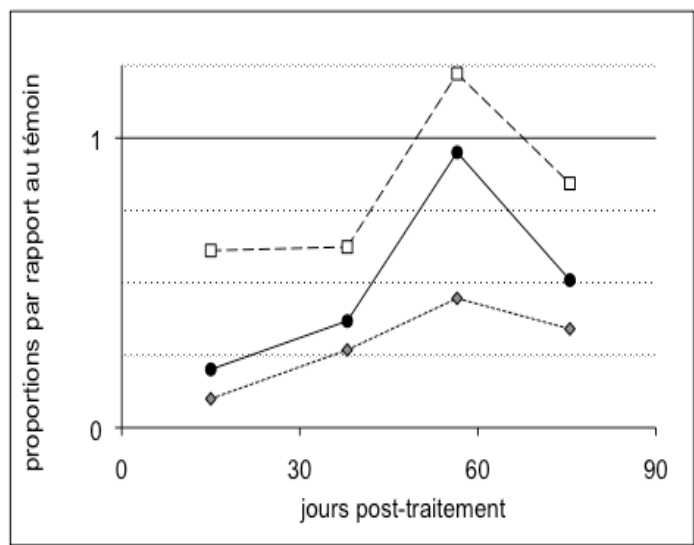

d

Figure 1 : Evolution des densités journalières et des proportions par rapport au témoin en fonction des jours après traitement. La Figure 1a montre l'évolution des densités dans le village en fonction du temps, évaluée à partir du nombre total de moustiques capturés dans la case sentinelle en l'absence de tout traitement. Les Figures $1 \mathrm{a}, \mathrm{b}, \mathrm{c}$ montrent l'évolution du rapport entre le nombre de An. gambiae s.l. capturée par rapport au témoin en relation avec les différents traitements (DEET, losange et pointillés ; KBR 3023 points et trait plein ; perméthrine, carrés et trait haché) en fonction du nombre de jours après imprégnation pour chacune des trois fractions de l'échantillon : nombre total (graphe a), gorgée (graphe b), et sous la moustiquaire (graphe c). 
A. BADOLO et al. / Int. J. Biol. Chem. Sci. 6(1): 237-247, 2012

Tableau 1 : Nombre $(\mathrm{N})$ et pourcentage de moustiques (\%) par méthode d'échantillonnage.

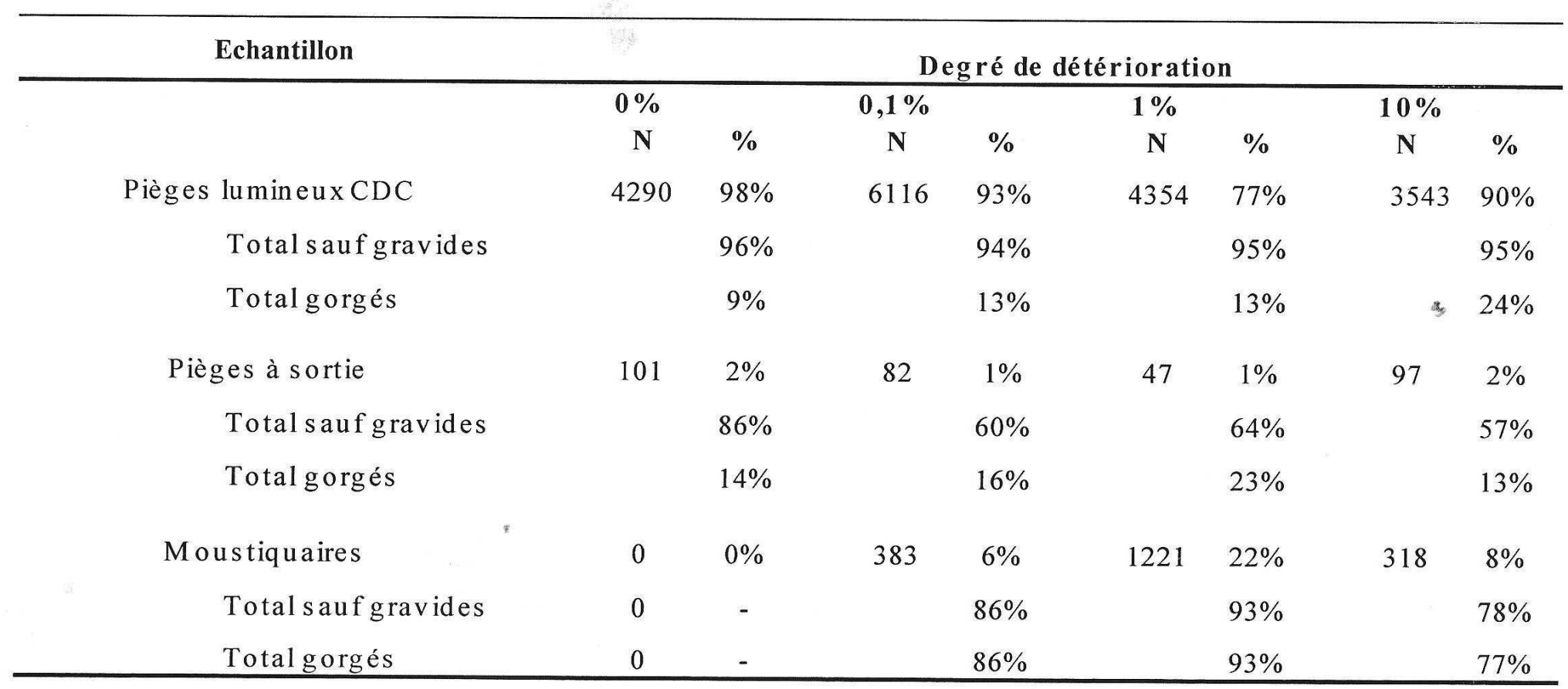

Le pourcentage est exprimé par rapport à l'ensemble de l'échantillon et pour chaque méthode, la contribution (\%) de chaque fraction de moustiques (total sauf gravides, gorgés, sous moustiquaire) est déterminé. 
A. BADOLO et al. / Int. J. Biol. Chem. Sci. 6(1): 237-247, 2012

Tableau 2 : Moyenne géométrique (MG) et intervalles de confiance à 95\% (entre parenthèses) du nombre de moustiques capturés.

\begin{tabular}{|c|c|c|c|c|c|c|c|c|c|}
\hline \multirow[t]{3}{*}{ Echantillon } & \multirow[t]{3}{*}{ Traitements } & \multicolumn{8}{|c|}{ Degréde détérioration } \\
\hline & & \multicolumn{2}{|l|}{$0 \%$} & \multicolumn{2}{|c|}{$0,1 \%$} & \multicolumn{2}{|l|}{$1 \%$} & \multicolumn{2}{|l|}{$10 \%$} \\
\hline \multirow{2}{*}{\multicolumn{10}{|c|}{ Total (sauf gravides) }} \\
\hline & & & & & & & & & \\
\hline & Té m o in & $\begin{array}{c}70,9 \\
(63.2-79.7)\end{array}$ & - & $\begin{array}{c}92,1 \\
\left(793^{3}-1069\right)\end{array}$ & & $\begin{array}{c}77,2 \\
(664-89.8)\end{array}$ & - & $\begin{array}{c}51,5 \\
(44,1-60,2)\end{array}$ & - \\
\hline & D E E T & $-(00,2-10$ & & $\begin{array}{c}37,9 \\
(31,3-45,7)\end{array}$ & $59 \%$ & $\begin{array}{c}32,0 \\
(26,3-39,0)\end{array}$ & $59 \%$ & $\begin{array}{c}34,5 \\
(29,5-40,2)\end{array}$ & $33 \%$ \\
\hline & K B R 3023 & - & & $\begin{array}{c}23,4 \\
(19,9-27,6)\end{array}$ & $75 \%$ & $\begin{array}{c}27,0 \\
(22,6-32,3)\end{array}$ & $65 \%$ & $\begin{array}{c}19,2 \\
(15,8-23,4)\end{array}$ & $63 \%$ \\
\hline & Perméthrine & - & & $\begin{array}{c}56,2 \\
(45,6-69,4)\end{array}$ & $39 \%$ & $\begin{array}{c}67,1 \\
(56,8-79,2)\end{array}$ & $13 \%$ & $\begin{array}{c}69,5 \\
(58,2-82,9)\end{array}$ & $0 \%$ \\
\hline \multicolumn{10}{|l|}{ Totalgorgés } \\
\hline & Tém o in & $\begin{array}{c}6,9 \\
(6,1-7,8)\end{array}$ & - & $\begin{array}{c}17,6 \\
(15,4-20,1)\end{array}$ & & $\begin{array}{c}27,2 \\
(23,6-31,3)\end{array}$ & - & $\begin{array}{c}14,5 \\
(12,7-17,6)\end{array}$ & - \\
\hline & D E E T & - & & $\begin{array}{c}7,7 \\
(6,4-9,1)\end{array}$ & $57 \%$ & $\begin{array}{c}8,1 \\
(6,4-10,2)\end{array}$ & $70 \%$ & $\begin{array}{c}9,8 \\
(8,1-11,9)\end{array}$ & $34 \%$ \\
\hline & K B R 3023 & - & & $\begin{array}{c}4,2 \\
(3,5-5,1)\end{array}$ & $76 \%$ & $\begin{array}{c}5,7 \\
(4,5-7,2)\end{array}$ & $79 \%$ & $\begin{array}{c}5,1 \\
(4,1-6,3)\end{array}$ & $66 \%$ \\
\hline & Perméthrine & - & & $\begin{array}{c}10,1 \\
(8,2-12,5)\end{array}$ & $43 \%$ & $\begin{array}{c}13,5 \\
(11,2-16,2)\end{array}$ & $50 \%$ & $\begin{array}{c}26,0 \\
(22,2-30,5)\end{array}$ & $0 \%$ \\
\hline \multicolumn{10}{|c|}{ Total moustiquaire } \\
\hline & Té m o in & $\begin{array}{l}0,0 \\
(-)\end{array}$ & - & $\begin{array}{c}4,3 \\
(3,7-5,0)\end{array}$ & & $\begin{array}{c}16,1 \\
(13,6-19,0)\end{array}$ & - & $\begin{array}{c}3,8 \\
(3,3-4,4)\end{array}$ & - \\
\hline & D E E T & & & $\begin{array}{c}0,8 \\
(0,6-1,1)\end{array}$ & $81 \%$ & $\begin{array}{c}2,1 \\
(1,4-2,9)\end{array}$ & $87 \%$ & $\begin{array}{c}0,6 \\
(0,4-0,9)\end{array}$ & $83 \%$ \\
\hline & K B R 3023 & & & $\begin{array}{c}0,3 \\
(0,2-0,4)\end{array}$ & $93 \%$ & $\begin{array}{c}1,5 \\
(1,0-2,0)\end{array}$ & $91 \%$ & $\begin{array}{c}0,4 \\
(0,2-0,6)\end{array}$ & $89 \%$ \\
\hline & Perméthrine & & & 0,0 & $100 \%$ & 0,0 & $100 \%$ & 0,0 & $100 \%$ \\
\hline
\end{tabular}

Ces valeurs sont en relation avec la surface de détérioration et la nature du traitement des moustiquaires pour les différentes fractions de l'échantillon global. La réduction entraînée par les traitements est exprimée en pourcentage de la moyenne par rapport au témoin. Les tirets remplacent des valeurs qui n'ont pu être déterminées. 


\section{DISCUSSION}

Les moustiquaires traitées dans leur ensemble entraînent une réduction du nombre de moustiques capturés par rapport au témoin pour une même surface endommagée. La réduction est plus importante pour le KBR 3023 que pour la perméthrine et le DEET. Pour un même niveau de détérioration, les moustiquaires imprégnées au KBR 3023 procurent une plus grande protection que celles imprégnées au DEET qui elles-mêmes sont plus efficaces que les moustiquaires témoins. L'agressivité est plus faible lorsque la surface détériorée est faible $(0,1 \%)$, elle est par contre élevée à $1 \%$. Port et Boreham (1982) et Charlwood (1986) ont démontré que l'utilisation des moustiquaires réduisait le nombre d'Anophelinae gorgés même si les moustiquaires sont déchirées. Toutefois, quand la surface de détérioration atteint 1,4\% de la surface, le nombre d'Anopheles arabiensis qui se gorgent n'est pas plus faible que dans la situation sans moustiquaires (Lines, 1996). Au niveau des moustiquaires à $10 \%$ de surface détériorée, on observe une tendance à la réduction de l'agressivité qui reflète plutôt une situation où le moustique peut atteindre facilement le dormeur sous la moustiquaire et ressortir aussi facilement, limitant la probabilité d'être pris par le piège lumineux CDC et la capture à l'intérieur de la moustiquaire. Au niveau des moustiquaires à $0,1 \%$, les moustiques atteignent plus difficilement le dormeur à cause de la petitesse des trous. Carnevale et al. (1992) avaient obtenu des réductions de l'agressivité lorsque les moustiquaires endommagées à $5 \%$ étaient imprégnées à la deltaméthrine mais la réduction de l'agressivité la plus élevée obtenue était de $56,1 \%$, valeur en moyenne plus faible que celles que nous avons obtenues avec le KBR 3023 et le DEET. Avec la perméthrine, nous obtenons une réduction en deçà et, lorsque la moustiquaire présente une surface de détérioration de $10 \%$, nous obtenons plutôt une augmentation du nombre de moustiques gorgés par rapport au témoin. Une explication pourrait être le mode d'action des différents traitements. En effet, le KBR
3023 et le DEET qui sont des répulsifs et éloignent les moustiques du dormeur, en témoigne la réduction du nombre de moustiques par rapport au témoin. La perméthrine pourrait avoir un faible effet répulsif, entrainant donc une faible réduction du nombre de moustiques. Il pourrait aussi s'agir d'une modification du comportement des moustiques par la perméthrine qui les dirigerait vers le piège CDC.

Ainsi, il apparaît que les répulsifs, en particulier le KBR 3023, soient plus indiqués que les pyréthrinoïdes dans l'imprégnation des moustiquaires endommagées. Mieux, utilisé sur des moustiquaires à faible surface endommagée $(0,1 \%)$, le KBR 3023 entraîne une plus grande réduction de l'agressivité de $90 \%$ comparé à la moustiquaire intacte non imprégnée. Snow et al. (1988) trouvaient que même intacte, la moustiquaire non imprégnée ne protégeait qu'à 90\% contre les piqûres des moustiques, ce qui justifie l'imprégnation des moustiquaires. $\mathrm{Si}$ la moustiquaire est détériorée, une imprégnation à l'aide d'un répulsif efficace et rémanent comme le KBR 3023 (Costantini et al., 2004) pourrait accroître son efficacité. L'imprégnation accrôit la rémanence des répulsifs à usage corporel et leur activité est plus prolongée que si le répulsif était utilisé en application corporelle. Curtis et al. (1987) ont obtenu avec des bandes imprégnées de DEET une protection pendant deux mois lorsque ces bandes n'étaient utilisées que deux heures par jour. Notre étude a duré trois mois et les moustiquaires sont restées accrochées pendant toute la durée de l'étude. Ces moustiquaires en plus de la protection personnelle, éloignent les moustiques de la maison. Ce qui témoignent la réduction du nombre total de moustiques obtenue avec les moustiquaires imprégnées de répulsifs.

Un des objectifs de cette étude était de déterminer l'usure limite pour qu'une moustiquaire ne puisse plus être utilisée. Les résultats ne nous ont pas permis de déterminer cette limite mais nous avons observé qu'une moustiquaire détériorée à plus de $1 \%$ a une faible efficacité et donc serait inutilisable si 
elle n'était pas imprégnée. Il est donc mieux pour garantir l'efficacité d'une moustiquaire qu'elle soit intacte, à défaut, l'imprégner à l'aide d'un répulsif pourrait accroître son efficacité.

\section{REMERCIEMENTS}

Cette étude a bénéficié d'un financement du programme d'évaluation des pesticides (WHOPES) de l'OMS. Nous remercions Dr. Morteza Zaim directeur du programme WHOPES pour son soutien. Merci à tout le personnel du Centre National de Recherche et de Formation sur le Paludisme (CNRFP) en particulier aux techniciens pour la collaboration. Enfin, nous sommes reconnaissant à tous les habitants du village de Goden pour leur coopération.

\section{REFERENCES}

Badolo A, Ilboudo-Sanogo E, Ouedraogo AP, Costantini C. 2004. Evaluation of the sensitivity of Aedes aegypti and Anopheles gambiae complex mosquitoes to two insect repellents: DEET and KBR 3023. Trop. Med. Int. Health., 9(3): 330334.

Carnevale P, Bitsindou P, Diomande L, Robert V. 1992. Insecticide impregnation can restore the efficiency of torn bed nets and reduce man-vector contact in malaria endemic areas. Trans. R. Soc. Trop. Med. Hyg., 86(4): 362-364.

Chandre F, Manguin S, Brengues C, Dossou Yovo J, Darriet F, Diabate A, Carnevale P, Guillet P. 1999. Current distribution of a pyrethroid resistance gene $(\mathrm{kdr})$ in Anopheles gambiae complex from west Africa and further evidence for reproductive isolation of the Mopti form. Parassitologia, 41(1-3): 319-322.

Charlwood JD. 1986. A differential response to mosquito nets by Anopheles and Culex mosquitoes from Papua New Guinea. Trans. R. Soc. Trop. Med. Hyg., 80(6): 958-960.

Chavasse D, Attawell K, Reed C. 1999. Insecticide Treated Net Projects: a Handbook for Managers. Malaria Consortium.
Corbel V, Stankiewicz M, Pennetier C, Fournier D, Stojan J, Girard E, Dimitrov M, Molgo J, Hougard JM, Lapied B. 2009. Evidence for inhibition of cholinesterases in insect and mammalian nervous systems by the insect repellent deet. BMC Biol., 7: 47.

Costantini C, Badolo A, Ilboudo-Sanogo E. 2004. Field evaluation of the efficacy and persistence of insect repellents DEET, IR3535, and KBR 3023 against Anopheles gambiae complex and other Afrotropical vector mosquitoes. Trans. R. Soc. Trop. Med. Hyg., 98(11): 644-652.

Costantini C, Li SG, Della Torre A, Sagnon N, Coluzzi M, Taylor CE. 1996. Density, survival and dispersal of Anopheles gambiae complex mosquitoes in a west African Sudan savanna village. Med. Vet. Entomol., 10(3): 203-219.

Curtis CF, Lines JD, Ijumba J, Callaghan A, Hill N, Karimzad MA. 1987. The relative efficacy of repellents against mosquito vectors of disease. Med. Vet. Entomol., 1(2): 109-119.

Edwards FW. 1941. Mosquitoes of the Ethiopian Region. III. Culicine Adults and Pupae. British Museum (Natural History): London.

Enayati A, Hemingway J. 2010. Malaria management: past, present, and future. Annu. Rev. Entomol., 55: 569-591.

Fanello C, Kolaczinski JH, Conway DJ, Carnevale P, Curtis CF. 1999. The kdr pyrethroid resistance gene in Anopheles gambiae: tests of non-pyrethroid insecticides and a new detection method for the gene. Parassitologia, 41(1-3): 323326.

Frances SP, Cooper RD, Sweeney AW. 1998. Laboratory and field evaluation of the repellents deet, CIC-4, and AI3-37220 against Anopheles farauti (Diptera: Culicidae) in Australia. J. Med. Entomol., 35(5): 690-693.

Gillies MT, Coetzee M. 1987. A supplement to the Anophelinae of Africa south of the Sahara (Afrotropical Region). The South African Institute for Medical Research. 
Ilboudo-Sanogo E, Cuzin-Ouattara N, Diallo DA, Cousens SN, Esposito F, Habluetzel A, Sanon S, Ouedraogo AP. 2001. Insecticide-treated materials, mosquito adaptation and mass effect: entomological observations after five years of vector control in Burkina Faso. Trans. R. Soc. Trop. Med. Hyg., 95(4): 353-360.

Lengeler C. 2004. Insecticide-treated bed nets and curtains for preventing malaria. Cochrane Database Syst. Rev., 2: CD000363.

Lines J. 1996. Review: mosquito nets and insecticides for net treatment: a discussion of existing and potential distribution systems in Africa. Trop. Med. Int. Health., 1(5): 616-632.

Macintyre K, Littrell M, Keating J, Hamainza B, Miller J, Eisele TP. 2011. Determinants of hanging and use of ITNs in the context of near universal coverage in Zambia. Health Policy Plan.

N'Guessan R, Corbel V, Akogbeto M, Rowland M. 2007. Reduced efficacy of insecticide-treated nets and indoor residual spraying for malaria control in pyrethroid resistance area, Benin. Emerg. Infect. Dis., 13(2): 199-206.

Nabarro DN, Tayler EM. 1998. The "roll back malaria" campaign. Science, 280(5372): 2067-2068.

Payne CD. 1987. The GLIM System : Generalised Linear Interactive Modelling System Manual. Numerical Algorithms Group: Oxford.

Pennetier C, Costantini C, Corbel V, Licciardi S, Dabire RK, Lapied B, Chandre F, Hougard JM. 2009. Synergy between repellents and organophosphates on bed nets: efficacy and behavioural response of natural free-flying $A n$. gambiae mosquitoes. PLoS ONE., 4(11): e7896.
Port GR, Boreham PFL. 1982. The effect of bed nets on feeding by Anopheles gambiae Giles (Diptera: Culicidae). Bull. Entomol. Res., 72(03): 483-488.

Qiu H, Jun HW, McCall JW. 1998. Pharmacokinetics, formulation, and safety of insect repellent N,N-diethyl-3methylbenzamide (deet): a review. J. Am. Mosq. Control Assoc., 14(1): 12-27.

Ranson H, Abdallah H, Badolo A, Guelbeogo WM, Kerah-Hinzoumbe C, YangalbeKalnone E, Sagnon N, Simard F, Coetzee M. 2009. Insecticide resistance in Anopheles gambiae: data from the first year of a multi-country study highlight the extent of the problem. Malar. J., 8: 299.

Robert V, Carnevale P. 1991. Influence of deltamethrin treatment of bed nets on malaria transmission in the Kou valley, Burkina Faso. Bull. World Health Organ., 69(6): 735-740.

Rozendaal JA. 1997. Vector Control: Methods for Use by Individuals and Communities. World Health Organization.

Rutledge LC, Collister DM, Meixsell VE, Eisenberg GH. 1983. Comparative sensitivity of representative mosquitoes (Diptera: Culicidae) to repellents. J. Med. Entomol., 20(5): 506-510.

Sharp BL, Ridl FC, Govender D, Kuklinski J, Kleinschmidt I. 2007. Malaria vector control by indoor residual insecticide spraying on the tropical island of Bioko, Equatorial Guinea. Malar. J., 6: 52.

Snow RW, Rowan KM, Lindsay SW, Greenwood BM. 1988. A trial of bed nets (mosquito nets) as a malaria control strategy in a rural area of The Gambia, West Africa. Trans. R. Soc. Trop. Med. Hyg., 82(2): 212-215. 